\title{
A RAD-based linkage map and comparative genomics in the gudgeons (genus Gnathopogon, Cyprinidae)
}

\author{
Ryo Kakioka ${ }^{1 *}$, Tomoyuki Kokita ${ }^{2}$, Hiroki Kumada ${ }^{2}$, Katsutoshi Watanabe ${ }^{1}$ and Noboru Okuda ${ }^{3}$
}

\begin{abstract}
Background: The construction of linkage maps is a first step in exploring the genetic basis for adaptive phenotypic divergence in closely related species by quantitative trait locus (QTL) analysis. Linkage maps are also useful for comparative genomics in non-model organisms. Advances in genomics technologies make it more feasible than ever to study the genetics of adaptation in natural populations. Restriction-site associated DNA (RAD) sequencing in next-generation sequencers facilitates the development of many genetic markers and genotyping. We aimed to construct a linkage map of the gudgeons of the genus Gnathopogon (Cyprinidae) for comparative genomics with the zebrafish Danio rerio (a member of the same family as gudgeons) and for the future QTL analysis of the genetic architecture underlying adaptive phenotypic evolution of Gnathopogon.

Results: We constructed the first genetic linkage map of Gnathopogon using a $198 \mathrm{~F}_{2}$ interspecific cross between two closely related species in Japan: river-dwelling Gnathopogon elongatus and lake-dwelling Gnathopogon caerulescens. Based on 1,622 RAD-tag markers, a linkage map spanning 1,390.9 cM with 25 linkage groups and an average marker interval of $0.87 \mathrm{cM}$ was constructed. We also identified a region involving female-specific transmission ratio distortion (TRD). Synteny and collinearity were extensively conserved between Gnathopogon and zebrafish.
\end{abstract}

Conclusions: The dense SNP-based linkage map presented here provides a basis for future QTL analysis. It will also be useful for transferring genomic information from a "traditional" model fish species, zebrafish, to screen candidate genes underlying ecologically important traits of the gudgeons.

\section{Background}

Ecological and phenotypic diversification in closely related species (or populations) provides an excellent opportunity for testing the role of natural selection in evolution [1]. Understanding the genetic architecture underlying such diversification is currently a fundamental topic in evolutionary ecology. Among the forwardgenetic approaches that are currently available to study natural populations, quantitative trait locus (QTL) mapping is a useful method. QTL mapping can be used to find the genetic basis of fitness-related traits of modest heritability, if the subject species can be bred and

\footnotetext{
* Correspondence: kakioka@terra.zool.kyoto-u.ac.jp; kokita@fpu.ac.jp 1 Department of Zoology, Graduate School of Science, Kyoto University, Kitashirakawa-Oiwake-cho, Sakyo-ku, Kyoto 606-8502, Japan

${ }^{2}$ Department of Marine Bioscience, Fukui Prefectural University, 1-1 Gakuen-cho, Obama 917-0003, Japan

Full list of author information is available at the end of the article
}

selected for the divergent phenotypes under controlled conditions [2]. Construction of a linkage map provides an essential basis for identifying chromosomal regions containing Mendelian single-gene traits and quantitative traits by genetic linkage analysis [2]. Linkage maps also serve as a link to the genomic information of model species and related non-model species by enabling genomic comparison, thus facilitate the discovery of candidate genes of non-model organisms [3-5].

Next-generation sequencers, or massively parallel sequencers, are making it more feasible to develop a large number of genetic markers, construct highly dense linkage maps, and practice comparative genomics. Thus, advances in genomics technologies make it more feasible than ever to explore the genetic basis of adaptation in both ecological model and non-model species [6]. In particular, restriction-site associated DNA (RAD) sequencing (RAD-seq) is readily available for non-model
Ciomed Central

(c) 2013 Kakioka et al.; licensee BioMed Central Ltd. This is an Open Access article distributed under the terms of the Creative Commons Attribution License (http://creativecommons.org/licenses/by/2.0), which permits unrestricted use, distribution, and reproduction in any medium, provided the original work is properly cited. 
organisms [7-9]. RAD-seq aims to explore singlenucleotide polymorphisms (SNPs) adjacent to restriction endonuclease sites. Through the massively parallel sequencing of DNA fragments flanking the restriction sites, or RAD tags, RAD-seq identifies SNPs and scores them as co-dominant markers. Hundreds or thousands of RAD-tag markers are obtained, and the markers are genotyped simultaneously for multiple individuals. RADseq has been successfully applied to various organisms for the construction of linkage maps $[10,11]$, QTL analysis [12,13], linkage disequilibrium analysis [14], comparative genomics [11], and genome assembly [15].

Lake fishes provide well-known examples of adaptive radiations as well as less species-rich but still illustrative examples of adaptive divergences [16]. The environmental distinctness of lakes as compared to rivers often drives adaptive evolutionary changes in lake colonisers' traits related to foraging and locomotion. Therefore, the lake ecosystem is a model system for examining the genetics of ecological and phenotypic diversification. Gudgeons of the genus Gnathopogon (Cyprinidae) are temperate freshwater fishes widely distributed in East Asia that live mostly in rivers and are associated with a benthivorous feeding style [12]. However, Gnathopogon caerulescens inhabits the ancient Lake Biwa in Japan, which harbours some endemic fishes that have evolved to adapt to its limnetic environment [17], showing a planktivorous feeding style $[18,19]$. This species markedly differs from river-dwelling Gnathopogon species in morphology, such as body depth, gill raker density, and barbel length. These differences are considered an evolutionary consequence of adaptive divergence caused by the divergent habitat and resource use [20]. Other limnetic Gnathopogon populations in several lakes show signs of parallel adaptive evolution within this genus [20,21]. However, the genetic basis for the phenotypic differentiation and parallelism remains completely unknown.

The aim of our present study is to construct a linkage map of Gnathopogon for comparative genomics, and for the future QTL analysis to elucidate the genetic basis of the morphological evolution of Gnathopogon in relation to adaptations to lake environments. We constructed an $\mathrm{F}_{2}$ interspecific cross between G. caerulescens and riverdwelling Gnathopogon elongatus. Using this pedigree, we constructed a linkage map using RAD-seq and searched for alleles with unusual segregation ratios in the progeny. We also tested for synteny and gene order with other model fishes, especially the "traditional" model species, zebrafish, which belongs to the same family as gudgeons.

\section{Methods}

\section{Study organisms and mapping family}

Gnathopogon elongatus is widely distributed in western to central Japan. It lives in rivers and ponds, feeding on zoobenthos and benthic algae [18,19]. Gnathopogon caerulescens is a relative of G. elongatus [21] endemic to Lake Biwa in Japan and feeds on zooplankton exclusively in pelagic waters $[18,19]$. These two species can be bred easily by artificial insemination [19]. A female G. elongatus collected from a small inlet of Lake Biwa, Shiga Prefecture, and a male G. caerulescens from Iba-naiko Lagoon (connected to Lake Biwa) were used as founders of an $F_{2}$ intercross. A single $F_{1}$ female and male sibling were then crossed once to generate one full-sib $\mathrm{F}_{2}$ family that was reared under controlled conditions.

A total of 198 full-sib $F_{2}$ progeny were sampled to construct a linkage map. Fin clips and muscle tissues were preserved in $99 \%$ ethanol at room temperature for several months for DNA samples. Following the collection of DNA samples, fish were fixed in $10 \%$ formalin. To determine sex, fixed fish were dissected, and their gonads were observed under a microscope.

\section{DNA extraction and RAD library construction}

Genomic DNA of founders and their $F_{2}$ progeny was extracted from the preserved samples using a DNeasy Blood \& Tissue Kit (Qiagen). The concentration of extracted DNA was determined using a spectrophotometer. DNA quality was analysed using agarose gel electrophoresis. Approximately $1 \mu \mathrm{g}$ of purified DNA was processed to obtain four RAD libraries each including 50 individuals. We followed the protocol of Etter [22] (see also [7]) and the instructions of the reagent manufacturers. In brief, genomic DNA from each individual was digested with the restriction endonuclease Sbfl (High fidelity; New England Biolabs). Modified Illumina adapters containing five nucleotides of barcode sequence (P1 adapters) unique to an individual in the library were ligated with T4 DNA ligase (New England Biolabs) to multiplex samples. The ligated DNA samples were pooled and sheared using a Covaris S-Series ultrasonicator (Covaris) into an average size of $500 \mathrm{bp}$. The sheared samples were size-selected to isolate DNA fragments spanning 300-500 bp by agarose gel electrophoresis. A Quick Blunting Kit (New England Biolabs) was used to convert $5^{\prime}$ or $3^{\prime}$ overhangs into phosphorylated blunt ends, and Klenow fragment (exo'; New England Biolabs) was then used to add adenine to the $3^{\prime}$ end. An adapter with divergent ends (P2 adapter) was ligated to enable selective PCR. The samples were amplified using Phusion High-Fidelity PCR Master Mix with HF Buffer (Finnzymes) by 18 cycles of PCR, and the libraries were finally purified with a MinElute column (Qiagen) to obtain approximately $20 \mu \mathrm{l}(12.3-31.3 \mathrm{ng} / \mu \mathrm{l})$ of sequencing libraries. PCR to the final purification was conducted twice for each pooled sample to create two sets of four libraries. The obtained RAD libraries were 
sequenced on an Illumina Genome Analyzer (GA) IIx in 75-bp single reads and the Illumina HiSeq 2000 in 100-bp single reads, each in four lanes of flow cells. The sequence dataset for this study was submitted to the Sequence Read Archive under accession number DRA000602.

\section{Genotyping}

Raw Illumina reads were filtered to discard those of low quality. Sequences with ambiguous barcode sequences were also eliminated from the subsequent marker processing using Stacks ver. 0.998 [23]. Sequences were first sorted to individuals according to the barcode sequences. Sequences from the Illumina HiSeq 2000 were truncated and analysed together with those from the Illumina GAIIx to increase read depth and overcome the PCR errors and biases in the sampling across alleles, loci, and individuals that are associated with nextgeneration sequencing [24]. To infer RAD loci, a minimum stack depth of 3 was required to create a stack, a maximum sequence mismatch of 2 was allowed between stacks to merge into a locus within an individual, and a maximum sequence difference of 3 was allowed to infer a homologous locus between parents. Genotypes were determined at the inferred RAD loci, requiring minimum stack depth of 20 to be called as homozygous, and correcting for the neglected heterozygote alleles due to their low coverage depth.

\section{Linkage map construction}

A linkage map was created using JoinMap 4.0 [25] for $\mathrm{F}_{2}$-type markers genotyped more than $85 \%$ of progeny. Markers showing significant segregation distortion $\left(\chi^{2}\right.$ test, $P<0.001$, d.f. $\left.=2\right)$ were excluded. Linkage groups were identified with an independence LOD threshold of 7. Unlinked markers and small linkage groups including less than 3 markers were excluded from further analysis. The linkage map was built using the regression mapping algorithm, a recombination frequency smaller than 0.4, and a LOD larger than 1. Up to three rounds of marker positioning were conducted with a jump threshold of 5 . A ripple was performed after the addition of each new marker. Map distances were calculated using Kosambi's mapping function. Following the initial mapping, potential errors that appear as doubtful double-recombinants were identified using genotype probabilities function of JoinMap $(P<0.001)$. The suspicious genotype was replaced by a missing value as suggested by Isidore et al. [26] and van Os et al. [27]. Then, a linkage map was constructed again using the corrected dataset. Potential error elimination and linkage map construction was iterated until no dubious genotype was identified, removing markers with $>20 \%$ missing value or that is distorted $\left(\chi^{2}\right.$ test, $P<0.001$, d.f. $=2$ ) in each iteration. The resultant linkage maps were drawn using MapChart ver. 2.2 [28]. We also estimated the corrected length of the linkage map by multiplying the length of each linkage group by $(m+1)$ / $(m-1)$, where $m$ is the number of markers in the linkage group [29]. The coverage of the genome by the linkage map was next estimated by calculating $c=1-e^{-2 d n / L}$, where $d$ is the average interval of markers, $n$ is the number of markers, and $L$ is the length of the linkage map [30].

\section{Analysis of transmission ratio distortion}

Technical artifacts may be responsible for the distorted markers, but biological processes known as transmission ratio distortion (TRD) also cause a deviation from Mendelian segregation [31,32]. First, to explore TRD, a linkage map was constructed without excluding distorted markers. Then, we compared linkage maps with and without distorted markers to find linkage groups with extensive differences in marker assignment. The comparison revealed a substantial difference in marker assignment in LG3 (see Results). Therefore, we further studied this linkage group regarding sex-specific TRD, which could be distinguished from segregation distortion due to artifacts. We sorted $F_{2}$ progeny by sex and constructed a linkage map for each group (LG3M for male progeny; homologous linkage group was not identified for female progeny due to extensive distortion) with the same condition as above, except the LOD threshold of 10 was used for clustering markers. Segregation of markers present on LG3M was $\chi^{2}$-tested $(\alpha=0.001$, d.f. $=2$ ) for the expected 1:2:1 segregation ratio, and the genotypic ratios of male and female progeny were plotted along LG3M to visualise the direct cause of the distortion.

\section{Sequence comparison}

Consensus sequences of the mapped RAD-tag markers (70 bases in length) were aligned with genomic sequences of four model fishes. The zebrafish Danio rerio ( $\mathrm{Zv} 9$ ), three-spined stickleback Gasterosteus aculeatus (ver. 1.0), medaka Oryzias latipes (ver. 1.0), and fugu Takifugu rubripes (ver. 5.0) genome sequences were downloaded, and blastn (BLAST+ ver. 2.2.26 [33]) searches with an $e$-value cutoff of $10^{-10}$ were conducted. In cases where the search of a query sequence hit two or more loci, a hit with the smallest $e$-value was considered significant; if the difference of the $e$-values between the first and the second smallest hits was not greater than $10^{3}$, the hit was considered insignificant. Significant hits on the chromo- somes were used, including unoriented scaffolds assigned to chromosomes in the fugu genome. The Oxford grids [34] were constructed to study synteny and to compare positions of the homologous loci using Grid Map ver. 3.0a (http://cbr.jic.ac.uk/dicks/software/Grid_Map/). 


\section{Results}

\section{RAD-tag sequencing and genotyping}

Illumina sequencing with GAIIx yielded a total of 142,563,874 75-base reads, and HiSeq 2000 sequencing yielded $697,482,400$ 100-base reads. The average count of RAD tags per individual was 3,842,573 (SD 966,921). RAD tags were aligned and clustered into 44,109 stacks (Additional file 1), and 11,463 candidate RAD loci were inferred. For the analysis of the $\mathrm{F}_{2}$ mapping population, 2,819 RAD-tag markers were informative and were scored for sufficient numbers of progeny. Among them, 1,887 markers were retained after discarding those with a deviation from a Mendelian segregation pattern, and then they were passed forward into the linkage map construction.

\section{Linkage map}

Linkage analysis identified 25 linkage groups (LG1LG25) containing a total of 1,622 markers (Table 1) after

Table 1 Summary of the sex-averaged map of Gnathopogon

\begin{tabular}{|c|c|c|c|}
\hline & $\begin{array}{l}\text { No. of } \\
\text { markers }\end{array}$ & $\begin{array}{l}\text { Length } \\
\text { (cM) }\end{array}$ & $\begin{array}{l}\text { Average marker } \\
\text { interval (cM) }\end{array}$ \\
\hline LG1 & 73 & 61.05 & 0.85 \\
\hline LG2 & 72 & 74.87 & 1.05 \\
\hline LG3 & 4 & 30.36 & 10.12 \\
\hline LG4 & 42 & 31.72 & 0.77 \\
\hline LG5 & 58 & 61.59 & 1.08 \\
\hline LG6 & 100 & 73.70 & 0.74 \\
\hline LG7 & 69 & 67.64 & 0.99 \\
\hline LG8 & 79 & 59.86 & 0.77 \\
\hline LG9 & 67 & 37.06 & 0.56 \\
\hline LG10 & 50 & 69.77 & 1.42 \\
\hline LG11 & 69 & 44.19 & 0.65 \\
\hline LG12 & 76 & 51.35 & 0.68 \\
\hline LG13 & 116 & 50.70 & 0.44 \\
\hline LG14 & 73 & 70.26 & 0.98 \\
\hline LG15 & 77 & 49.10 & 0.65 \\
\hline LG16 & 98 & 61.45 & 0.63 \\
\hline LG17 & 71 & 50.84 & 0.73 \\
\hline LG18 & 68 & 66.51 & 0.99 \\
\hline LG19 & 74 & 55.67 & 0.76 \\
\hline LG20 & 59 & 55.81 & 0.96 \\
\hline LG21 & 50 & 68.35 & 1.39 \\
\hline LG22 & 40 & 31.62 & 0.81 \\
\hline LG23 & 64 & 61.65 & 0.98 \\
\hline LG24 & 41 & 47.30 & 1.18 \\
\hline LG25 & 32 & 58.46 & 1.89 \\
\hline Average & 64.88 & 55.64 & \\
\hline Total & 1884 & $1,858.36$ & 0.87 \\
\hline
\end{tabular}

the removal of dubious genotypes. The sex-averaged map spanned 1,390.9 cM, with a mean distance between markers of $0.87 \mathrm{cM}$ (Figure 1 and Additional file 2: Figure S1). The lengths of linkage groups ranged from 30.4 (LG3) to $74.9 \mathrm{cM}$ (LG2); the number of markers mapped on a linkage group ranged from 4 (LG3) to 116 (LG13). The corrected length of the linkage map was estimated at 1,455.1 cM, which is converted to a genome coverage of $86.9 \%$.

\section{Transmission ratio distortion in female progeny}

A total of 2,627 markers, including distorted ones, were assigned to 25 linkage groups. Each of them had homologous relation to one of the linkage groups identified without distorted markers. LG3D, the homologous linkage group of LG3, showed apparent differences in marker assignment: only 4 markers were mapped on LG3, whereas 120 markers were mapped on LG3D (Additional file 3: Figure S2). This was considered to be due to TRD on LG3; we therefore constructed a linkage map for LG3M to further study TRD on LG3. LG3 contained four markers over 30.4 cM; LG3M contained 81 markers over 66.9 cM (Additional file 3: Figure S2; Figure 2A).

Many of the markers assigned to LG3M genotyped in male progeny yielded larger $X^{2}$-test $P$ values for the expected Mendelian 1:2:1 segregation in the $F_{2}$ intercross than the threshold applied $(\alpha=0.001$, d.f. $=2)$, with a median of 0.11 (range $1.1 \times 10^{-3}-6.7 \times 10^{-1}$; Figure $2 \mathrm{~B}$ ). In contrast, most of the same markers genotyped in female progeny yielded smaller $\chi^{2}$-test $P$ values, with a median of $8.2 \times 10^{-13}$ (range $8.9 \times 10^{-21}-4.0 \times 10^{-4}$; Figure $2 \mathrm{~B}$ ), and only one marker exceeded the threshold. There was a trend for the female $\chi^{2}$-test $P$ value to decrease toward one end of the hypothetical homologous linkage group. TRD in female progeny was mainly due to the lack of homozygote alleles derived from the grandmother (Figure 2C). Such trend was not apparent for male progeny (Figure 2D).

\section{Syntenic relationship between Gnathopogon and model fish species}

BLAST searches of the 1,622 mapped Gnathopogon RAD-tag marker consensus sequences against the genome sequences of zebrafish, stickleback, medaka, and fugu indicated variation in the syntenic relationship between Gnathopogon and the respective species. Homology was most frequently inferred to the zebrafish genome, with $30.3 \%$ of Gnathopogon sequences being mapped to it. In contrast, the other three species yielded limited numbers of similarity hits. Only 3.7\%, 2.8\%, and $2.8 \%$ of Gnathopogon sequences mapped to the stickleback, medaka, and fugu genome sequences, respectively. Of the hits against the zebrafish genome, 97.1\% aligned 
to the chromosomes that had a one-to-one relationship with Gnathopogon linkage groups, suggesting highly conserved synteny between Gnathopogon and zebrafish (Figure 3). Because of the small number of significant hits, synteny between Gnathopogon and the three fish species was inconclusive.

Thirteen syntenic pairs of linkage groups and chromosomes (LG/chromosome 1, 4, 5, 6, 9, 11, 12, 19, 21, $22,23,24$, and 25) showed apparent collinear relation- ships between Gnathopogon and zebrafish (Figure 4 and Additional file 4: Figure S3), whereas other pairs exhibited disrupted collinearity, suggesting intrachromosomal rearrangements (Figure 4B and Additional file 4: Figure S3). Thus, in addition to synteny, gene order within a syntenic chromosome was also inferred to be substantially retained between Gnathopogon and zebrafish.

Although only two of four markers on LG3 were aligned to the zebrafish chromosome 3, 24 of 25 markers

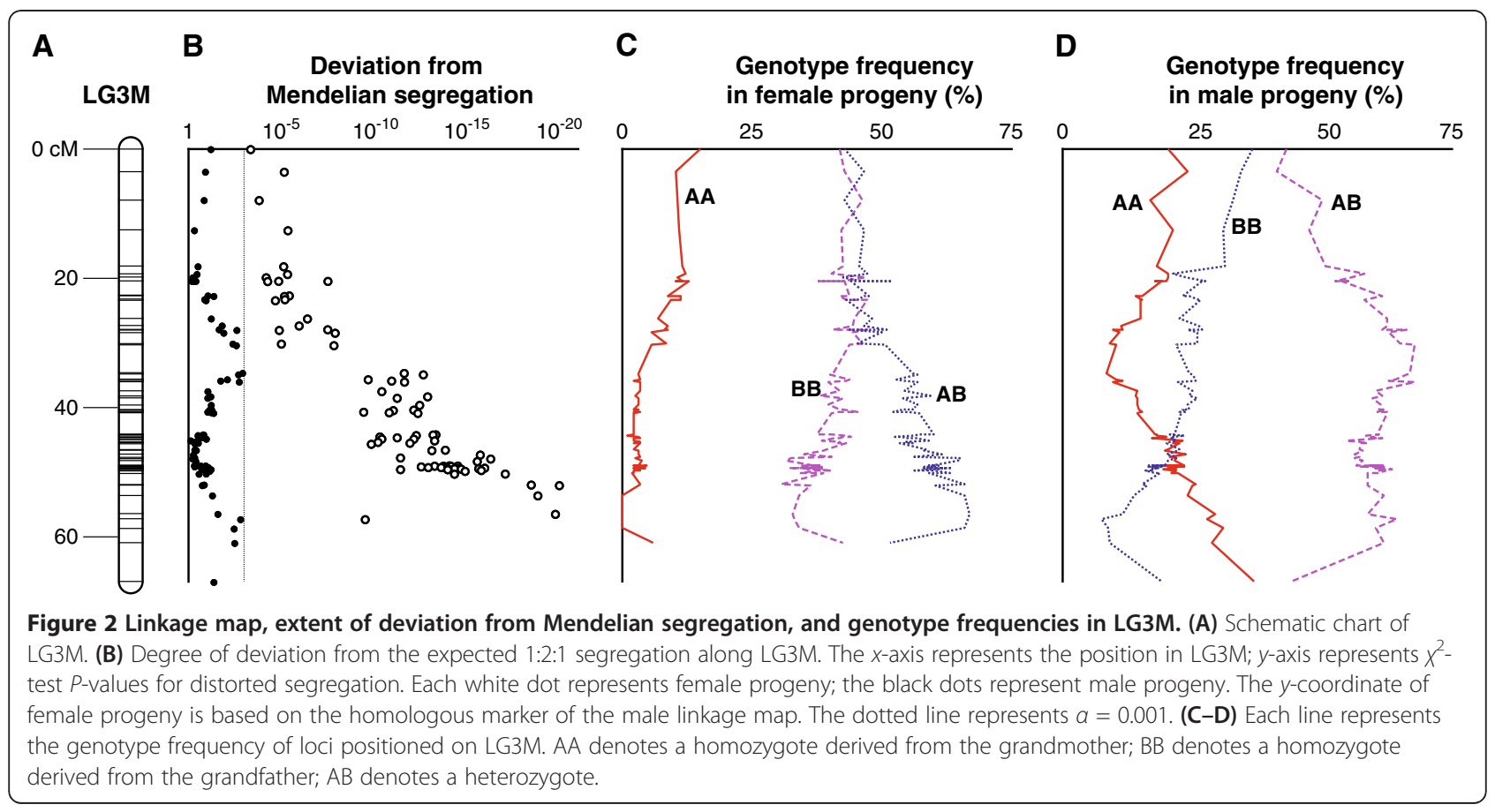




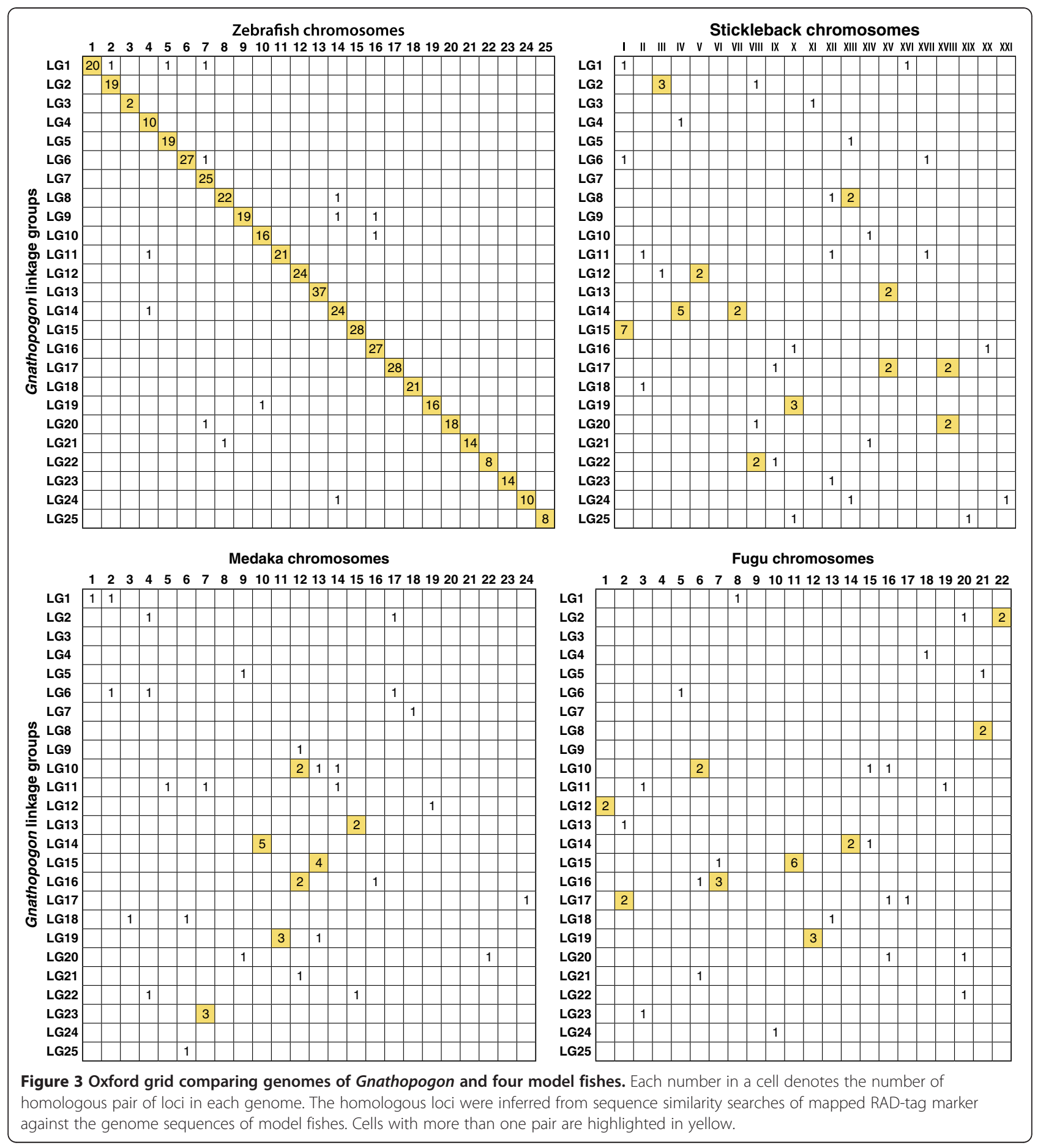

with significant hits on LG3M showed a synteny of LG3M with zebrafish chromosome 3 and collinearity of loci (Figure 5). This result further supports not only a syntenic relationship between LG3 and zebrafish chromosome 3 but also a successful mapping of LG3M, which seems to have experienced a limited influence of TRD.

\section{Discussion}

RAD sequencing and linkage mapping

Here, we present the first linkage map of Gnathopogon, which is also the first for the Gobioninae, a diverse group of fishes within the family Cyprinidae. Taking advantage of massively parallel sequencers, we obtained a high-density linkage map with 25 linkage groups and an 


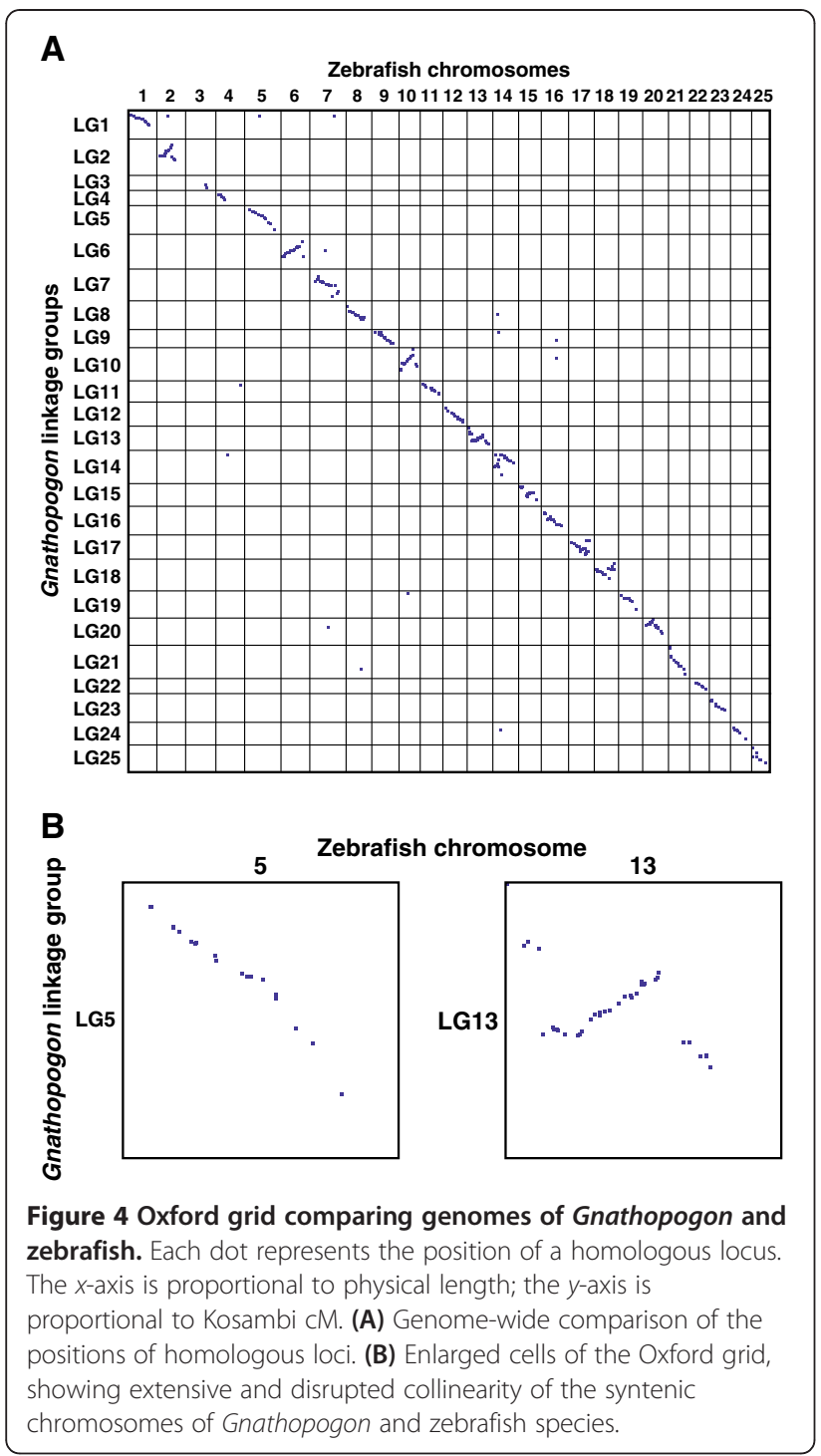

average marker distance of approximately $0.87 \mathrm{cM}$ that covers $86.9 \%$ of the genome. The number of identified linkage groups is congruent with the karyotypes of $G$. caerulescens $(2 n=50)$ and G. elongatus $(2 n=50)$ [35]. Such a dense linkage map contains detailed information on the genomic structure of an organism and is therefore useful for studies involving comparative genomics and QTL mapping.

To date, AFLP and microsatellite markers have been popular options for linkage analyses in organisms without genomic information. Although AFLP markers require no prior information about the genome of a target species, they are anonymous dominant markers bearing no sequence information for genomic comparison; microsatellite markers are sequence-based, but they are costly and time-consuming if hundreds or thousands of markers are involved. Our linkage map is solely based on RAD-seq. In contrast to AFLP and microsatellite markers, RAD-tag markers have advantages for the genomic analysis of non-model organisms. These markers are sequence-based, allowing the practice of comparative genomics [10,11], which aids in exploring candidate genes for traits of interest [13] and even assembling de novo genomic sequences [15]. Moreover, allelic information on a large number of markers is readily available without prior curation and labourious experiments. The present study further demonstrates the utility of RADseq in the genomic study of a non-model organism, yielding a wealth of genomic information without prior knowledge of the genome of a subject species.

\section{Sex-specific TRD}

We found a female-specific TRD in the alleles of marker loci homologous to LG3M. TRD refers to a phenomenon in which the alleles of a locus of a heterozygous parent are not transmitted equally, resulting in deviation from the Mendelian 1:1 segregation [32,36].

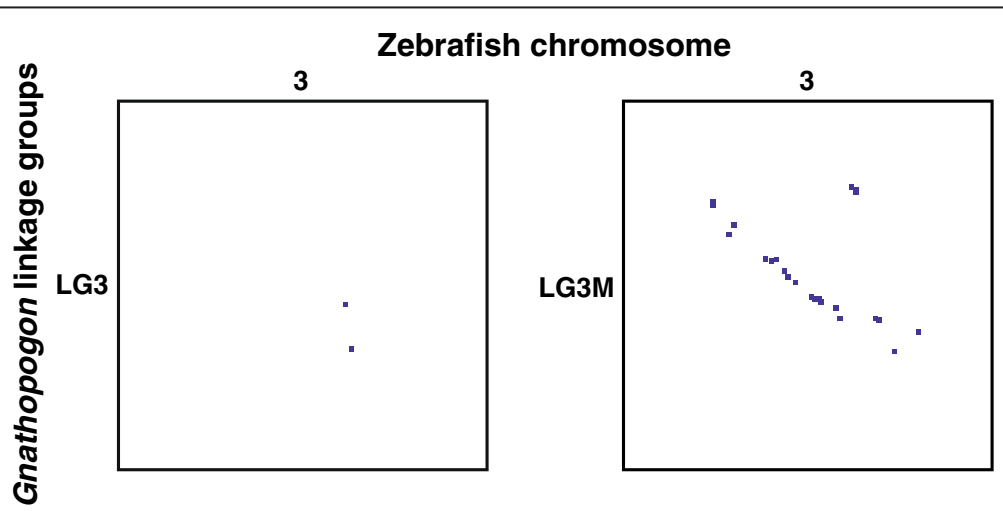

Figure 5 Oxford grid comparing Gnathopogon linkage groups LG3 and LG3M and zebrafish chromosome 3. Each dot represents the position of a homologous locus. The $x$-axis is proportional to physical length; the $y$-axis is proportional to Kosambi cM. 
This phenomenon is an extension of segregation distortion, referring to the unequal segregation during meiosis; TRD also includes cases in which postmeiotic effects or unknown causes yield distorted transmission of alleles. The extent of TRD is also correlated with genomic divergence, which is empirically shown as the abundance of distorted markers in interspecific crosses relative to intraspecific crosses [37-39]. The divergence time of the two Gnathopogon species used to construct the mapping family is estimated at 4 million years ago (mya) [21]. This might have caused substantial differences between the genomes of the two species, due to the accumulated genomic changes following the divergence. Taken together, the genomic data suggest that TRD occurred in the interspecific cross of the Gnathopogon lineages. To test this hypothesis, intraspecific crosses of Gnathopogon species would be needed.

Sex-specific TRD occurs in several animals [40-42]. A study of female-specific TRD in the mouse Mus musculus suggested that the TRD was caused by the post-fertilisation reduction of female viability that involved a specific region of a chromosome [40]. The female-specific TRD in Gnathopogon also seems to be due to postzygotic causes, such as the reduced viability of the female embryo or fry involving a deleterious gene on LG3 or a deleterious gene regulated by a gene on LG3. This gene might be a recessive lethal allele derived from the female founder, G. elongatus. Male viability might not be reduced because the lethality of hybrids is rescued by a gene in the male-determining region. This explanation seems likely because male offspring exhibited no such TRD on LG3M and because the allele frequency in female progeny exhibited the trend along LG3M. Further studies are needed to elucidate the mechanism of TRD by investigating the survivability of gametes and zygotes and the allele transmission using interspecific and intraspecific crosses.

TRD can affect the transmission of alleles in the hybrid zone. In mapping populations of the iris Iris fulva and Iris brevicaulis, for example, TRD causes an asymmetric introgression of alleles of I. fulva [43], which is attributable to the more frequent introgression of I. fulva alleles into $I$. brevicaulis in the natural hybrid zones between the iris species [44]. Our subject species, G. caerulescens and G. elongatus, show parapatric distribution in the Lake Biwa basin. G. elongatus inhabits the tributaries, lagoon, and shallow littoral zone of the lake. Conversely, G. caerulescens inhabits the offshore limnetic zone. However, G. caerulescens spawns in the lagoon and littoral zone, and the reproductive seasons of these species overlap, resulting in reproductive season sympatry the coexistence during the reproductive season [19]. These species occasionally hybridise in natural habitats, that is, the premating barrier is incomplete ([19], Kokita, unpublished data). The TRD might contribute to reproductive barriers between sympatric Gnathopogon species by lowering the fitness of hybrids because hybrid individuals produce a smaller number of viable offspring [45].

\section{Genomic comparison}

There was high synteny between Gnathopogon and zebrafish. Majority of the RAD loci located on a Gnathopogon linkage group are colocalised to a single zebrafish chromosome. Considering the old divergence of the lineages leading to each species, which date back to 117 mya (95\% CI, 100-135 mya) [46], this is a substantial conservation. It is therefore likely that extensive interchromosomal rearrangements have not occurred in either of the lineages leading to Gnathopogon and zebrafish since they diverged. This conclusion supports the findings from the comparative analysis of genomic structure among fish and mammalian species indicate that interchromosomal rearrangements are less frequent in teleost fishes than in mammals [47-50]. Collinearity was also general between Gnathopogon and zebrafish, yet interruptions of collinearity were not rare. These data suggest that intrachromosomal rearrangements, such as inversions, occurred in either or both of the two lineages after the divergence of their ancestors.

Lineages including Gnathopogon, zebrafish, or the common carp Cyprinus carpio are major lineages within Cyprinidae that diverged in the early stage of the diversification of cyprinid fishes [46,51]. Cyprinid fishes show great cytogenetic variation. Their chromosome numbers range from $2 n=42$ (Acheilognathus gracilis [52], or $2 n=30$ if the taxonomically controversial Paedocypris carbunculus is placed within Cyprinidae [53]) to $2 n=$ 417-470 (Ptychobarbus dipogon [54,55]), with a mode at $2 n=50$, followed by $2 n=48$ [56]. Thus, it has been suggested that the ancestral karyotype of cyprinid fishes was $2 n=48-50$, and that polyploidisation occurred in several groups within the Cyprinidae [57-63]. Genomescale syntenic analyses between zebrafish and other cyprinid fishes have been conducted for common carp $(2 n=100)[64]$ and grass carp Ctenopharyngodon idella $(2 n=48)$ [65], both of which revealed some cases of interchromosomal rearrangements. The majority of the linkage groups in common carp have two-to-one relationships with zebrafish chromosomes, suggesting tetraploidisation in the common carp lineage. Those analyses also revealed a common carp linkage group sharing loci with two zebrafish chromosomes, which is speculated to have resulted from a chromosome recombination or transposition followed by fusion of homologous chromosomes during the process of diploidisation following tetraploidisation [64]. Most grass carp linkage groups are syntenic with zebrafish chromosomes, many of which have one-to-one relationships. One grass carp 
linkage group exhibits a one-to-two relationship with zebrafish chromosomes, suggesting chromosomal fusion. In grass carp, substantial macrosynteny and several cases of interchromosomal rearrangements are suggested. Considering the macrosynteny between Gnathopogon and zebrafish and the substantially straightforward trace of autopolyploidisation in the genome of common carp, the ancestral karyotype of Cyprinidae seems to be $2 n=$ 50 , concordant with the inference from the comparative karyological studies $[62,63]$. On the other hand, syntenic analysis between zebrafish and Mexican cave tetra Astyanax mexicanus (Characidae; $2 n=50$ ) revealed cases of putative interchromosomal rearrangements [3], such that syntenic loci of an Astyanax linkage group resided on several zebrafish chromosomes. These were suggested to be caused by gene duplications after the divergence of the lineages 248 mya (95\% CI, 227-268 mya) [66]. Nevertheless, the analysis also revealed that synteny was conserved between Astyanax and zebrafish in numerous genomic regions. Combining the syntenic relationships and the genomic information of zebrafish, candidate genes for ecologically and evolutionarily important traits were identified in Astyanax [3].

Cyprinidae is the largest family of freshwater fishes. They have highly diverse morphology, ecology, and physiology, which are adapted to the vast range of habitats and resources they exploit $[55,67]$. Evolutionary ecological studies have been conducted in various cyprinid species concerning, e.g., adaptive radiation [68], hybridisation $[69,70]$, and resource polymorphism [71]. However, the genomic basis and consequences of their diversification have not been extensively explored. In this study, Gnathopogon are suggested to be able to take advantage of the genomic information of a model cyprinid species, zebrafish, and its conserved synteny and collinearity with Gnathopogon. They may provide a prediction of candidate genes responsible for the traits related to phenotypic divergence that have ecological and evolutionary significance. Conservation of synteny and collinearity might be expected among cyprinid fishes, which could be advantageous for transferring genomic information between species [3-5,72]. This raises the prospect that evolutionary genomic studies of cyprinid fishes are accelerated by the interspecific exchange of information and by complementary studies between species.

\section{Conclusions}

We constructed a highly dense linkage map of gudgeons (Gnathopogon) using RAD-seq. This map covers a majority of the genome, and the number of linkage groups is consistent with the haploid chromosome number of Gnathopogon. Sex-specific departure from a Mendelian inheritance pattern was identified in a linkage group. Synteny and collinearity are highly conserved between
Gnathopogon and the traditional model organism zebrafish. We inferred that extensive interchromosomal rearrangements are not common between Gnathopogon and zebrafish, but intrachromosomal rearrangements have occurred. This linkage map clarifies the genetic architecture underlying the morphological diversification of Gnathopogon using the future QTL analysis. The transfer of genomic information from zebrafish to Gnathopogon, enabled by their conserved synteny and collinearity, is also useful for screening candidate genes responsible for the traits of interest.

\section{Additional files}

Additional file 1: List of RAD stack sequences, including consensus sequences of RAD loci.

Additional file 2: Figure S1. A detailed linkage map of the interspecific cross between Gnathopogon caerulescens and Gnathopogon elongatus. The lengths of the linkage groups are based on Kosambi cM.

Additional file 3: Figure S2. Linkage maps of LG3, LG3M, and LG3D and extent of deviation from Mendelian segregation in LG3D. (A) Linkage maps of LG3, LG3M, and LG3D. The map distance estimates are in Kosambi cM. The homology of loci between linkage groups is indicated with a line. (B) Degree of deviation from the expected 1:2:1 segregation along LG3D. The $x$-axis represents the position on LG3D; $y$-axis represents $x^{2}$-test $P$-values for distorted segregation. The dotted line represent $a=0.001$.

Additional file 4: Figure S3. Comparison of syntenic pairs of a linkage group of Gnathopogon and a chromosome of zebrafish. Each dot represents the position of a homologous locus. The $x$-axis is proportional to physical length; the $y$-axis is proportional to Kosambi cM.

\section{Competing interests}

The authors declare that they have no competing interests.

\section{Authors' contributions}

$\mathrm{TK}$ and $\mathrm{NO}$ initiated the research project on trophic polymorphism in Gnathopogon. RK, TK, KW, and NO conceived and designed the experiments. RK, TK, and HK performed the experiments. RK conducted data analysis. RK and TK wrote the paper. All authors read and approved the final manuscript.

\section{Acknowledgements}

We are grateful to K. Kikuchi for valuable comments on the manuscript. We thank M. Nemoto for providing a G. caerulescens founder for the mapping family. This study was partly supported by Grants-in-Aid from the Ministry of Education, Culture, Sports, Science and Technology of Japan (No. 20370009 and No. 23657019) and "Formation of a Strategic Base for Biodiversity and Evolutionary Research: from Genome to Ecosystem" of the Global COE Program A06.

\section{Author details}

'Department of Zoology, Graduate School of Science, Kyoto University, Kitashirakawa-Oiwake-cho, Sakyo-ku, Kyoto 606-8502, Japan. ${ }^{2}$ Department of Marine Bioscience, Fukui Prefectural University, 1-1 Gakuen-cho, Obama 917-0003, Japan. ${ }^{3}$ Center for Ecological Research, Kyoto University, 509-3, Hirano 2-chome, Otsu 520-2113, Japan.

Received: 24 July 2012 Accepted: 14 January 2013

Published: 16 January 2013

\section{References}

1. Schluter D: The Ecology of Adaptive Radiation. Oxford: Oxford University Press; 2000.

2. Ellegren $H$, Sheldon BC: Genetic basis of fitness differences in natural populations. Nature 2008, 452:169-175. 
3. Gross JB, Protas M, Conrad M, Scheid PE, Vidal O, Jeffery WR, Borowsky R, Tabin CJ: Synteny and candidate gene prediction using an anchored linkage map of Astyanax mexicanus. Proc Natl Acad Sci USA 2008, 105:20106-20111.

4. Sarropoulou E, Fernandes JM: Comparative genomics in teleost species: Knowledge transfer by linking the genomes of model and non-model fish species. Comp Biochem Physiol Part D Genomics Proteomics 2011, 6:92-102.

5. Sarropoulou E, Nousdili D, Magoulas A, Kotoulas G: Linking the genomes of nonmodel teleosts through comparative genomics. Marine Biotechnol 2008, 10:227-233.

6. Stapley J, Reger J, Feulner PG, Smadja C, Galindo J, Ekblom R, Bennison C, Ball AD, Beckerman AP, Slate J: Adaptation genomics: the next generation. Trends Ecol Evol 2010, 25:705-712.

7. Etter PD, Bassham S, Hohenlohe PA, Johnson EA, Cresko WA: SNP discovery and genotyping for evolutionary genetics using RAD sequencing. Methods Mol Biol 2011, 772:157-178.

8. Davey JW, Blaxter ML: RADSeq: next-generation population genetics. Brief Funct Genomics 2010, 9:416-423.

9. Baird NA, Etter PD, Atwood TS, Currey MC, Shiver AL, Lewis ZA, Selker EU, Cresko WA, Johnson EA: Rapid SNP discovery and genetic mapping using sequenced RAD markers. PLoS One 2008, 3:e3376.

10. Ingvarsson PK, Baxter SW, Davey JW, Johnston JS, Shelton AM, Heckel DG, Jiggins CD, Blaxter ML: Linkage mapping and comparative genomics using next-generation RAD sequencing of a non-model organism. PLOS One 2011, 6:e19315.

11. Amores A, Catchen J, Ferrara A, Fontenot Q, Postlethwait JH: Genome evolution and meiotic maps by massively parallel DNA sequencing: spotted gar, an outgroup for the teleost genome duplication. Genetics 2011, 188:799-808.

12. Pfender WF, Saha MC, Johnson EA, Slabaugh MB: Mapping with RAD (restriction-site associated DNA) markers to rapidly identify QTL for stem rust resistance in Lolium perenne. Theor Appl Genet 2011, 122:1467-1480

13. Chutimanitsakun Y, Nipper RW, Cuesta-Marcos A, Cistue L, Corey A, Filichkina T, Johnson EA, Hayes PM: Construction and application for QTL analysis of a Restriction Site Associated DNA (RAD) linkage map in barley. BMC Genomics 2011, 12:4.

14. Hohenlohe PA, Bassham S, Currey M, Cresko WA: Extensive linkage disequilibrium and parallel adaptive divergence across threespine stickleback genomes. Philosophical Transactions of the Royal Society B: Biological Sciences 2012, 367:395-408.

15. The Heliconius Genome Consortium, Dasmahapatra KK, Walters JR, Briscoe AD, Davey JW, Whibley A, Nadeau NJ, Zimin AV, Hughes DS, Ferguson LC, et al: Butterfly genome reveals promiscuous exchange of mimicry adaptations among species. Nature 2012, doi:10.1038/nature11041.

16. Hendry AP: Ecological speciation! Or the lack thereof? Can J Fish Aquat Sci 2009, 66:1383-1398.

17. Rossiter A: Lake Biwa as a topical ancient lake. In Advances in Ecological Research Volume 31. Edited by Rossiter A, Kawanabe H. London: Academic Press; 2000:571-598.

18. Hosoya K: Gnathopogon. In Freshwater Fishes of Japan. 3rd edition. Edited by Kawanabe H, Mizuno N, K H. Tokyo: Yama-Kei Publishers; 2001:297-299.

19. Nakamura M: Gnathopogon. In Cyprinid Fishes of Japan. Tokyo: Research Institute for Natural Resources; 1969:112-125.

20. Hosoya K: Tamoroko-zoku gyorui no keito to keishitsu-chikan (Phylogeny and character displacement in Gnathopogon fishes). In Nippon no TansuiGyorui: Sono Bumpu, Heni, Shubunka o Megutte (Freshwater Fishes in Japan: Their Distribution, Variation and Speciation). Edited by Mizuno N, Goto A. Tokyo: Tokai University Press; 1987:31-40.

21. Kakioka R, Kokita T, Tabata R, Mori S, Watanabe K: The origins of limnetic forms and cryptic divergence in Gnathopogon fishes (Cyprinidae) in Japan. Environ Biol Fishes 2012, doi:10.1007/s10641-012-0054-x.

22. Etter PD: Sequenced RAD markers for rapid SNP discovery and genetic mapping, ver. 2; [https://www.wiki.ed.ac.uk/display/RADSequencing/Home].

23. Catchen JM, Amores A, Hohenlohe P, Cresko W, Postlethwait JH: Stacks: building and genotyping loci de novo from short-read sequences. G3: Genes, Genomes, Genetics 2011, 1:171-182.

24. Davey JW, Hohenlohe PA, Etter PD, Boone JQ, Catchen JM, Blaxter ML: Genome-wide genetic marker discovery and genotyping using nextgeneration sequencing. Nat Rev Genet 2011, 12:499-510.
25. Van Ooijen JW: JoinMap ${ }^{\circledR}$, Software for the calculation of genetic linkage maps in experimental populations. Kyazma B.V.: Wageningen; 2006.

26. Isidore E, van Os H, Andrzejewski S, Bakker J, Barrena I, Bryan GJ, Caromel B, van Eck H, Ghareeb B, de Jong W, et al: Toward a marker-dense meiotic map of the potato genome: lessons from linkage group I. Genetics 2003, 165:2107-2116

27. van Os H, Stam P, Visser RG, van Eck HJ: SMOOTH: a statistical method for successful removal of genotyping errors from high-density genetic linkage data. Theor Appl Genet 2005, 112:187-194.

28. Voorrips RE: MapChart: software for the graphical presentation of linkage maps and QTLs. J Hered 2002, 93:77-78.

29. Chakravarti A, Lasher LK, Reefer JE: A maximum likelihood method for estimating genome length using genetic linkage data. Genetics 1991, 128:175-182.

30. McDaniel SF, Willis JH, Shaw AJ: A linkage map reveals a complex basis for segregation distortion in an interpopulation cross in the moss Ceratodon purpureus. Genetics 2007, 176:2489-2500.

31. Naumova A, Greenwood C, Morgan K: Imprinting and deviation from Mendelian transmission ratios. Genome 2001, 44:311-320.

32. Montagutelli $X$, Turner $\mathrm{R}$, Nadeau JH: Epistatic control of non-Mendelian inheritance in mouse interspecific crosses. Genetics 1996, 143:1739-1752.

33. Camacho C, Coulouris G, Avagyan V, Ma N, Papadopoulos J, Bealer K, Madden T: BLAST+: architecture and applications. BMC Bioinforma 2009, 10:421.

34. Edwards JH: The Oxford Grid. Ann Hum Genet 1991, 55:17-31.

35. Ueno K, Ye Y-J, Umeoka T: A comparative study of chromosomes in the cyprinid fish genera Gnathopogon and Squalidus of Japan. Nippon Suisan Gakkaishi 1992, 58:1273-1277.

36. Crow JF: Why is Mendelian segregation so exact? BioEssays 1991, 13:305-312.

37. Hall MC, Willis JH: Transmission ratio distortion in intraspecific hybrids of Mimulus guttatus: implications for genomic divergence. Genetics 2005, 170:375-386.

38. Jenczewski E, Gherardi M, Bonnin I, Prosperi JM, Olivieri I, Huguet T: Insigh on segregation distortions in two intraspecific crosses between annual species of Medicago (Leguminosae). Theor Appl Genet 1997, 94:682-691.

39. Taylor D, Ingvarsson P: Common features of segregation distortion in plants and animals. Genetica 2003, 117:27-35.

40. Shendure J, Melo J, Pociask K, Derr R, Silver L: Sex-restricted nonMendelian inheritance of mouse chromosome 11 in the offspring of crosses between C57BL/6 J and (C57BL/6 J x DBA/2 J)F1 mice. Mamm Genome 1998, 9:812-815.

41. Routtu J, Jansen B, Colson I, De Meester L, Ebert D: The first-generation Daphnia magna linkage map. BMC Genomics 2010, 11:508.

42. Aparicio JM, Ortego J, Calabuig G, Cordero PJ: Evidence of subtle departures from Mendelian segregation in a wild lesser kestrel (Falco naumanni) population. Heredity 2010, 105:213-219.

43. Tang S, Okashah RA, Knapp SJ, Arnold ML, Martin NH: Transmission ratio distortion results in asymmetric introgression in Louisiana Iris. BMC Plant Biol 2010, 10:48

44. Cruzan MB, Arnold ML: Assortative mating and natural-selection in an Iris hybrid zone. Evolution 1994, 48:1946-1958.

45. Moyle LC, Graham EB: Genome-wide associations between hybrid sterility QTL and marker transmission ratio distortion. Mol Biol Evol 2006, 23:973-980.

46. Saitoh K, Sado T, Doosey MH, Bart HL Jr, Inoue JG, Nishida M, Mayden RL, Miya M: Evidence from mitochondrial genomics supports the lower Mesozoic of South Asia as the time and place of basal divergence of cypriniform fishes (Actinopterygii: Ostariophysi). Zoological Journal of the Linnean Society 2011, 161:633-662.

47. Kai W, Kikuchi K, Fujita M, Suetake H, Fujiwara A, Yoshiura Y, Ototake M, Venkatesh B, Miyaki $K$, Suzuki Y: A genetic linkage map for the tiger pufferfish, Takifugu rubripes. Genetics 2005, 171:227-238.

48. Jaillon O, Aury JM, Brunet F, Petit JL, Stange-Thomann N, Mauceli E, Bouneau L, Fischer C, Ozouf-Costaz C, Bernot A, et al: Genome duplication in the teleost fish Tetraodon nigroviridis reveals the early vertebrate proto-karyotype. Nature 2004, 431:946-957.

49. Kasahara M, Naruse K, Sasaki S, Nakatani Y, Qu W, Ahsan B, Yamada T, Nagayasu Y, Doi K, Kasai $Y$, et al: The medaka draft genome and insights into vertebrate genome evolution. Nature 2007, 447:714-719.

50. Kai W, Kikuchi K, Tohari S, Chew AK, Tay A, Fujiwara A, Hosoya S, Suetake $H$, Naruse K, Brenner S, et al: Integration of the genetic map and genome assembly of fugu facilitates insights into distinct features of genome evolution in teleosts and mammals. Genome Biol Evol 2011, 3:424-442. 
51. Mayden RL, Chen WJ, Bart HL, Doosey MH, Simons AM, Tang KL, Wood RM, Agnew MK, Yang L, Hirt MV, et al: Reconstructing the phylogenetic relationships of the earth's most diverse clade of freshwater fishesorder Cypriniformes (Actinopterygii: Ostariophysi): a case study using multiple nuclear loci and the mitochondrial genome. Mol Phylogenet Evol 2009, 51:500-514.

52. Hong YH, Zhou T: Studies on the karyotype and C-banding patterns in Acheilognathus gracilis with a discussion on the evolution of acheilognathid fifishes. Acta Genetica Sinica 1985, 12:143-148.

53. Liu S, Hui TH, Tan SL, Hong Y: Chromosome evolution and genome miniaturization in minifish. PLoS One 2012, 7:e37305.

54. Yu XY, Yu XJ: A schizothoracine fish species, Diptychus dipogon, with a very high number of chromosomes. Chromosome Information Service 1990, 48:17-18.

55. Catalog of Fishes electronic version (14 July 2011). [http://research. calacademy.org/research/ichthyology/catalog/fishcatmain.asp].

56. Arai R: Fish Karyotypes: A Check List. Tokyo: Springer; 2011.

57. Leggatt RA, Iwama GK: Occurrence of polyploidy in the fishes. Reviews in Fish Biology and Fisheries 2003, 13:237-246.

58. Le Comber SC, Smith C: Polyploidy in fishes: patterns and processes. Biol J Linn Soc 2004, 82:431-442.

59. Suzuki A: Chromosomal evolution of the suborder Cyprinoidei, with special reference to topical problems of phylogenetic relationships of their families. Cytobios 1996, 87:169-179.

60. Mank JE, Avise JC: Phylogenetic conservation of chromosome numbers in Actinopterygiian fishes. Genetica 2006, 127:321-327.

61. Cataudella S, Sola L, Muratori RA, Capanna E: The chromosomes of 11 species of cyprinidae and one cobitidae from Italy, with some remarks on the problem of polyploidy in the cypriniformes. Genetica 1977, 47:161-171.

62. Yu X, Zhou T, Li K, Li Y, Zhou M: On the karyosystematics of cyprinid fishes and a summary of fish chromosome studies in China. Genetica 1987, 72:225-236

63. Arai R: A chromosome study on two cyprinid fishes, Acrossocheilus labiatus and Pseudorasbora pumila pumila, with notes on eurasian cyprinids and their karyotypes. Bulletin of the National Science Museum Series A, Zoology 1982, 8:131-152.

64. Zheng X, Kuang Y, Zhang X, Lu C, Cao D, Li C, Sun X: A genetic linkage map and comparative genome analysis of common carp (Cyprinus carpio L.) using microsatellites and SNPs. Mol Genet Genomics 2011, 286:261-277.

65. Xia JH, Liu F, Zhu ZY, Fu J, Feng J, Li J, Yue GH: A consensus linkage map of the grass carp (Ctenopharyngodon idella) based on microsatellites and SNPs. BMC Genomics 2010, 11:135.

66. Nakatani M, Miya M, Mabuchi K, Saitoh K, Nishida M: Evolutionary history of Otophysi (Teleostei), a major clade of the modern freshwater fishes: Pangaean origin and Mesozoic radiation. BMC Evol Biol 2011, 11:177.

67. Winfield IJ, Nelson JS: Cyprinid Fishes: Systematics, Biology and Exploitation. London: Chapman \& Hall; 1991

68. de Graaf M, Dejen E, Osse JWM, Sibbing FA: Adaptive radiation of Lake Tana's (Ethiopia) Labeobarbus species flock (Pisces, Cyprinidae). Mar Freshw Res 2008, 59:391-407.

69. DeMarais BD, Dowling TE, Douglas ME, Minckley WL, Marsh PC: Origin of Gila seminuda (Teleostei: Cyprinidae) through introgressive hybridization: implications for evolution and conservation. Proc Natl Acad Sci USA 1992, 89:2747-2751.

70. Gerber AS, Tibbets CA, Dowling TE: The role of introgressive hybridization in the evolution of the Gila robusta complex (Teleostei: Cyprinidae). Evolution 2001, 55:2028-2039.

71. Komiya T, Fujita S, Watanabe K: A novel resource polymorphism in fish driven by differential bottom environments: an example from an ancient lake in Japan. PLoS One 2011, 6:e17430.

72. Christoffels A, Bartfai R, Srinivasan H, Komen H, Orban L: Comparative genomics in cyprinids: common carp ESTs help the annotation of the zebrafish genome. BMC Bioinforma 2006, 7(5):S2.

\section{doi:10.1186/1471-2164-14-32}

Cite this article as: Kakioka et al:: A RAD-based linkage map and comparative genomics in the gudgeons (genus Gnathopogon, Cyprinidae). BMC Genomics 2013 14:32.

\section{Submit your next manuscript to BioMed Central and take full advantage of:}

- Convenient online submission

- Thorough peer review

- No space constraints or color figure charges

- Immediate publication on acceptance

- Inclusion in PubMed, CAS, Scopus and Google Scholar

- Research which is freely available for redistribution 